\title{
Bridging the gap in knowledge between dyspnoea scientists and clinicians
}

\author{
Dan Adler ${ }^{1,2}$ \\ Affiliations: ${ }^{1}$ Division of Pulmonary Diseases, Geneva University Hospitals, Geneva, Switzerland. ${ }^{2}$ Dept of \\ Internal Medicine Specialties, Geneva Medical School, Geneva, Switzerland. \\ Correspondence: Dan Adler, Division of Pulmonary Diseases, Geneva University Hospitals, 4-6 Rue Gabrielle- \\ Perret-Gentil, 1211 Geneva 14, Switzerland. E-mail: dan.adlerahcuge.ch
}

@ERSpublications

A study in the ERJ suggests that dyspnoea per se is a major clinical endpoint in a chain of events leading to physical disability http://ow.ly/QGEg30l6gfj

Cite this article as: Adler D. Bridging the gap in knowledge between dyspnoea scientists and clinicians. Eur Respir J 2018; 52: 1801308 [https://doi.org/10.1183/13993003.01308-2018].

Dyspnoea can be defined as "a subjective experience of breathing discomfort that consists of qualitatively distinct sensations that vary in intensity" [1]. It derives from interactions among multiple physiological, psychological, social and environmental factors, and may induce secondary physiological and behavioural responses. The current definition of dyspnoea highlights the importance of the multidimensional nature of dyspnoea (sensory-perceptual, affective distress and impact domains), which in turn has led to the development of multidimensional tools to assess dyspnoea $[2,3]$. Our current understanding of dyspnoea has been fostered by the ongoing efforts of a community of scientists focused on the multidisciplinary/ translational aspects of dyspnoea research. As a result of continuous education, in which the European Respiratory Society was also involved, clinicians caring for these patients in daily practice finally agreed that dyspnoea is a neutral word to "hide" major respiratory suffering in individual patients [4]. Indeed, chronic breathlessness is very often compared with chronic pain and shares many neurophysiological similarities $[5,6]$.

Translational research suggests that when pathophysiological factors related to lung disease have been corrected, novel therapeutic approaches related to the brain integration of afferent information and the affective impact of dyspnoea may be a worthwhile path of study [7, 8]. For instance, the concept of dyspnoea-pain counterirritation, defined as the attenuation of pre-existing pain by intercurrent, experimentally induced dyspnoea, has well established neurophysiological correlates. By expanding the very same concept from the bench to the bedside, DANGers et al. [9] were able to demonstrate that relieving dyspnoea by noninvasive ventilation in patients with amyotrophic lateral sclerosis (ALS) was associated with decreased pain thresholds. This observation suggests that patients with ALS may become more susceptible to pain after initiation of noninvasive ventilation. A benefit on both dyspnoea and pain can also be achieved with pharmacological treatment targeting central opioid receptors. Currently, administration of low-dose opioids is the only evidence-based treatment to reduce chronic breathlessness [10]. Finally, by analogy with a recent approach in pain research, the modulation of breathing perception through virtual reality seems to be a new and promising research avenue $[11,12]$.

By contrast, classical clinical research has mainly focused on treating underlying pathophysiological dysfunction as the target of an intervention, rather than dyspnoea itself. This has met with conflicting results as most underlying pathological processes, such as emphysema or fibrosis, are not reversible. Why scant attention has been given to dyspnoea itself in clinical research is surprising, since it is a point of convergence for and the final path of most chronic respiratory diseases. However, evidence that dyspnoea 
is a major clinical endpoint remains scarce, although it has been shown to be an important predictor of mortality in the general population [13] and is a frequent symptom in all hospitalised patients [14]. Moreover, dyspnoea is a better predictor of mortality than forced expiratory volume in $1 \mathrm{~s}$ in patients with chronic obstructive pulmonary disease (COPD) [15]. Recent evidence also suggests that dyspnoea is an independent predictor of readmission or death in a selected group of COPD patients surviving respiratory failure, even after multiple adjustments for major comorbidities [16]. Thus, a robust demonstration that dyspnoea per se has a major impact on clinical outcome was needed to bridge this gap of knowledge between dyspnoea scientists and clinicians.

The important study by RAMON et al. [17] in this issue of the European Respiratory Journal (ERJ) has taken up the challenge of putting together "expert opinion" on the widely accepted concept of the dyspnoeainactivity vicious circle and real-world data obtained from two international, large-scale COPD cohorts (PAC-COPD [18] and ICE COLD ERIC [19]). First, they identified nine previously published conceptual models of the dyspnoea-inactivity vicious circle based on expert opinion. Using fitted equation models, the authors were able to demonstrate that none of the current models showed an appropriate fit with real-world data, although all of them replicated known associations between variables selected for the dyspnoeainactivity vicious circle. Second, they developed a comprehensive new vicious circle model using a limited number of candidate variables from the PAC-COPD cohort by the inclusion of biologically plausible variables and the stepwise exclusion of variables that did not improve model fit. Third, they validated the new model in the ICE COLD ERIC cohort to ascertain generalizability of the model in a second COPD cohort.

RAMON et al. [17] should be congratulated for this report, which is of major importance and will certainly stimulate the COPD community to design new/early interventions targeting dyspnoea as a specific endpoint in order to break the vicious circle. As always, there are some limitations but these are adequately addressed in the discussion section of the paper. The relatively short follow-up period of both cohorts may have restricted the ability to identify important variables (such as age) related to the vicious circle. Another limitation of the study is the use of the modified Medical Research Council (mMRC) scale to assess dyspnoea. Although the mMRC and the New York Heart Association share the same construct and are commonly accepted measures of dyspnoea, both tools are in fact measures of disability related to dyspnoea and not measures of dyspnoea itself [20]. This makes the argument of an association between dyspnoea and physical activity a bit circular. Therefore, the selection of specific tools to assess dyspnoea will be a necessary step for further research in the field. It is also unfortunate that "airway obstruction", rather than "dyspnoea", was the entry criterion in the dyspnoea-inactivity vicious circle. Having "dyspnoea" first in the circle would have increased the scope of the study to other important groups of patients: 1) symptomatic current or former smokers with preserved pulmonary function [21, 22]; 2) COPD and non-COPD patients with multimorbidity [23, 24]; and 3) frail older patients with respiratory and nonrespiratory diseases [25]. However, the authors clearly state that the study population was restricted to COPD patients and therefore, their results cannot be generalised to patients with other chronic respiratory diseases. Lastly, patients lost to follow-up may have had an impact on the selection of important variables in the final dyspnoea-inactivity model, given that they had a worse functional status at baseline and were those who died during follow-up. The authors argue that from a clinical point of view, only patients who survive COPD without severe comorbidities are those who present at clinical practice with features of the vicious circle.

However, with those limitations in mind, the study by RAMON et al. [17] has the major merit of bridging an important gap in our knowledge by identifying dyspnoea as a major clinical endpoint in a chain of events leading to major disability. The ERJ has already demonstrated its commitment in supporting high-quality, multidisciplinary, translational research [26-28], of which this study is a fine example that will serve to stimulate clinicians and researchers to design and test novel approaches targeting dyspnoea in order to change patients' health trajectories.

Conflict of interest: None declared.

\section{References}

1 Parshall MB, Schwartzstein RM, Adams L, et al. An official American Thoracic Society statement: update on the mechanisms, assessment, and management of dyspnea. Am J Respir Crit Care Med 2012; 185: 435-452.

2 Meek PM, Banzett R, Parsall MB, et al. Reliability and validity of the multidimensional dyspnea profile. Chest 2012; 141: 1546-1553.

3 Yorke J, Swigris J, Russell A-M, et al. Dyspnea-12 is a valid and reliable measure of breathlessness in patients with interstitial lung disease. Chest 2011; 139: 159-164.

4 Başoğlu M. Effective management of breathlessness: a review of potential human rights issues. Eur Respir J 2017; 49: 1602099.

5 Morélot-Panzini C, Demoule A, Straus C, et al. Dyspnea as a noxious sensation: inspiratory threshold loading may trigger diffuse noxious inhibitory controls in humans. J Neurophysiol 2007; 97: 1396-1404. 

2009; 48: 200-206

$7 \quad$ Morélot-Panzini C. Fooling the brain to alleviate dyspnoea. Eur Respir J 2017; 50: 1701383.

8 Similowski T. Treat the lungs, fool the brain and appease the mind: towards holistic care of patients who suffer from chronic respiratory diseases. Eur Respir J 2018; 51: 1800316.

9 Dangers L, Laviolette L, Georges M, et al. Relieving dyspnoea by non-invasive ventilation decreases pain thresholds in amyotrophic lateral sclerosis. Thorax 2017; 72: 230-235.

10 Ekström M, Nilsson F, Abernethy AA, et al. Effects of opioids on breathlessness and exercise capacity in chronic obstructive pulmonary disease. A systematic review. Ann Am Thorac Soc 2015; 12: 1079-1092.

11 Adler D, Herbelin B, Similowski T, et al. Reprint of "Breathing and sense of self: Visuo-respiratory conflicts alter body self-consciousness". Respir Physiol Neurobiol 2014; 204: 131-137.

12 Allard E, Canzoneri E, Adler D, et al. Interferences between breathing, experimental dyspnoea and bodily self-consciousness. Sci Rep 2017; 7: 9990.

13 Pesola GR, Ahsan H. Dyspnea as an independent predictor of mortality. Clin Respir J 2016; 10: $142-152$.

14 Stevens JP, Dechen T, Schwartzstein R, et al. Prevalence of dyspnea among hospitalized patients at the time of admission. J Pain Symptom Manage 2018.

15 Nishimura K, Izumi T, Tsukino M, et al. Dyspnea is a better predictor of 5-year survival than airway obstruction in patients with COPD. Chest 2002; 121: 1434-1440.

16 Dupuis Lozeron E, Soccal PM, Janssens J-P, et al. Severe dyspnea is an independent predictor of readmission or death in COPD patients surviving acute hypercapnic respiratory failure in the ICU. Front Med 2018; 5: 163.

17 Ramon MA, Ter Riet G, Carsin A-E, et al. The dyspnoea-inactivity vicious circle in COPD: development and external validation of a conceptual model. Eur Respir J 2018; 52: 1800079.

18 Garcia-Aymerich J, Gómez FP, Benet M, et al. Identification and prospective validation of clinically relevant chronic obstructive pulmonary disease (COPD) subtypes. Thorax 2011; 66: 430-437.

19 Strassmann A, Frei A, Haile SR, et al. Commonly used patient-reported outcomes do not improve prediction of copd exacerbations: a multicenter 41/2 year prospective cohort study. Chest 2017; 152: 1179-1187.

20 Bestall J, Paul E, Garrod R, et al. Usefulness of the Medical Research Council (MRC) dyspnoea scale as a measure of disability in patients with chronic obstructive pulmonary disease. Thorax 1999; 54: 581-586.

21 Woodruff PG, Barr RG, Bleecker E, et al. Clinical significance of symptoms in smokers with preserved pulmonary function. N Engl J Med 2016; 374: 1811-1821.

22 Regan EA, Lynch DA, Curran-Everett D, et al. Clinical and radiologic disease in smokers with normal spirometry. JAMA Intern Med 2015; 175: 1539-1549.

23 Vanfleteren LE, Spruit MA, Franssen FM. Tailoring the approach to multimorbidity in adults with respiratory disease: the NICE guideline. Eur Respir J 2017; 49: 1601696.

24 Adler D, Pépin J-L, Dupuis-Lozeron E, et al. Comorbidities and subgroups of patients surviving severe acute hypercapnic respiratory failure in the intensive care unit. Am J Respir Crit Care Med 2017; 196: $200-207$.

25 Bousquet J, Dinh-Xuan AT, Similowski T, et al. Should we use gait speed in COPD, FEV 1 in frailty and dyspnoea in both? Eur Respir J 2016; 48: 315-319.

26 Herigstad M, Faull OK, Hayen A, et al. Treating breathlessness via the brain: changes in brain activity over a course of pulmonary rehabilitation. Eur Respir J 2017; 50: 1701029.

27 Abdallah SJ, Wilkinson-Maitland C, Saad N, et al. Effect of morphine on breathlessness and exercise endurance in advanced COPD: a randomised crossover trial. Eur Respir J 2017; 50: 1701235.

28 Farver-Vestergaard I, O’Toole MS, O’Connor M, et al. Mindfulness-based cognitive therapy in COPD: a cluster randomised controlled trial. Eur Respir J 2018; 51: 1702082. 